\title{
El ocaso de los libros de texto como fuente de información entre los estudiantes de medicina
}

\author{
Constanza Ralph ${ }^{1}$, Arnoldo Riquelme ${ }^{2}$, Jorge A. Carvajal ${ }^{*}$
}

Resumen: Introducción: El proceso de enseñanza de la medicina ha cambiado a través de la historia en la medida que sus estudiantes cambian. Los profesores de medicina se adaptan con dificultades a los nuevos estudiantes y muchas veces no son capaces de reconocer los nuevos intereses de sus propios alumnos. En este contexto resulta importante analizar qué métodos de estudio utilizan los actuales alumnos durante los siete años de la carrera de Medicina. Métodos: Estudio basado en metodología mixta: cualitativa (grupales focales) y cuantitativa (encuestas) aplicadas a alumnos de quinto, sexto o séptimo año de la carrera, médicos recién egresados y docentes activos de la Escuela de Medicina de la Pontificia Universidad Católica. Resultados: Los resultados de nuestro estudio muestran que los métodos de estudio que más utilizan los estudiantes de medicina son: apuntes de clases (1-5 años) y los manuales (internado). Los encuestados creen que los manuales son muy importantes, que facilitan el aprendizaje, que deben estar actualizados y que de preferencia deben estar disponibles en formato digital. Los docentes usaron como método preferente de estudio, durante la carrera de medicina, los libros de texto. Conclusión: Los estudiantes de medicina han cambiado, y así han evolucionado sus técnicas de estudio/ aprendizaje. Es indispensable que los métodos docentes se adapten a estas nuevas características; por lo que es indispensable ayudar a los profesores a modernizar sus técnicas docentes adaptándolas a las necesidades de los alumnos.

Palabras claves: Manuales; Libros de texto; Generación XY Z; baby boomers; métodos de estudio; estudiantes de medicina.

Abstract: Introduction: The process of teaching medicine has changed throughout history as its students change. The medical professors adapt with difficulties to the new students and many times they are not able to recognize the new interests of its own students. In this context, it is important to analyze what methods of study the current students use during the seven years of the medical career. Methods: This study was based on a mixed methodology: qualitative (focus groups) and quantitative (surveys) applied to students in the fifth, sixth or seventh year of the medical studies, recently graduated doctors and active teachers of the Pontificia Universidad Católica de Chile. Results: The results of our study show that the study methods most used by medical students are: class notes (1-5 years) and handbooks (internship). Respondents believe that handbooks are very important, that they facilitate learning, that they should be up to date and that they should preferably be available in digital format. The teachers used as a preferred method of study, during the medical career, textbooks. Conclusion: Medical students have changed, and so have their study / learning techniques evolved. It is essential that teaching methods adapt to these new characteristics; thus, it is essential to help teachers to modernize their teaching techniques by adapting them to the needs of students.

Keywords: Handbooks; Textbooks; XY Z Generations; Baby boomers; Study methods; Medical students.

Fecha de envío: 17 de enero de 2017 - Fecha de aceptación: 27 de junio de 2017

\section{Introducción}

El proceso de enseñanza/aprendizaje de la medicina ha cambiado a través de la historia. La enseñanza tradicional de la medicina se ceñía al concepto de la tabula rasa (vernota *), el profesor guiaba la enseñanza como un maestro a seguir, y el proceso se centraba en la disciplina. Desde los años 50-60 en adelante, con el surgimiento de teorías y modelos de didáctica, la enseñanza se transforma en un proceso profesional, obligando a los docentes a prepararse en métodos pedagógicos y por ende la enseñanza se centra en el profesor. De modo más reciente, con el advenimiento de la sociedad del conocimiento, en que se desarrolla el acceso a la información para todos, la enseñanza de la medicina ha trocado a un modelo centrado en el alumno, donde el rol del profesor es estimular el aprendizaje autorregulado (Daura, 2011; Ponce, 2014).

(1) División de Obstetricia y Ginecología. Unidad de Medicina Materno Fetal

(2) Departamento de Gastroenterología. Escuela de Medicina, Pontificia Universidad Católica de Chile

Autor de Correspondencia:jcarva@med.puc.cl 
El proceso de enseñanza/aprendizaje de la medicina también ha evolucionado, de la mano del cambio generacional de sus estudiantes. Los alumnos que hoy cursan medicina o están recién egresados, nacieron en su gran mayoría a fines de los años 90 . Son representantes de lo que se ha llamado la generación $Z$, entendiendo por tal los nacidos desde 1995 en adelante. Todos los estudiantes de la generación $Z$ son nativos digitales (personas nacidas desde 1980 en adelante), este grupo de estudiantes, caracterizados por tener amplio acceso a internet, no conciben la vida sin avances tecnológicos, desarrollan múltiples tareas al mismo tiempo y privilegian la calidad de vida por sobre las responsabilidades (Silva et al., 2006). Sin duda poseen métodos de estudio diferentes que los de las generaciones anteriores; usan las nuevas tecnologías para sociabilizar pero en un porcentaje no menor, hasta en un $70 \%$, lo utilizan para realizar tareas escolares o universitarias (Han et al., 2014).

El impacto del cambio generacional en estudiantes de medicina ha sido reconocido como una dificultad, en la formación de médicos y especialistas (Kunakov, 2011; Money et al., 2014); los alumnos de hoy buscan priorizar su tiempo libre y pasar menos tiempo estudiando; el uso de internet pasó de ser algo novedoso a una herramienta más para las actividades diarias de estudio (Popescu \& Navarro, 2005). El rol del profesor se hace complejo, pues no solo debe captar las necesidades de aprendizaje de los alumnos, sino que debe entender sus métodos de aprendizaje, cautivándolos con sus modelos de enseñanza, pero a la vez estimulando el estudio auto dirigido.

El problema es que los profesores de medicina no son parte de la generación $Z$, sino que son parte de la generación de los baby boomers (nacidos entre 1945-1964) o de la generación $X$ (nacidos entre 1965-1981), y muy pocos de la generación $Y$ (nacidos entre 1982-1994), ocasionando que el entendimiento y la mirada de las nuevas tecnologías sea diferente a la de los alumnos. Muchos médicos docentes no se conectan bien con la generación actual de alumnos, valoran la productividad y no toleran el ocio, siguen pensando que lo más importante son las clases presenciales (cátedras) y el uso de libros de texto. Los profesores hablan una lengua anticuada, no se conectan con el lenguaje de la tecnología y no son capaces de reconocer los nuevos intereses de sus propios alumnos (Prensky, 2001; Kunakov, 2011).

Los profesores más jóvenes se han adaptado al proceso digital (inmigrantes digitales), pero mantienen gestos propios de su generación; muchos profesores no usan las plataformas online de los cursos, imprimen sus correos electrónicos, creen que los alumnos deben leer libros de texto y no aceptan el uso de computadores o tabletas digitales durante las clases, pues los alumnos parecen distraídos. El proceso de enseñanza/aprendizaje desde inmigrantes digitales a nativos digitales es sin duda difícil, y muchas veces los alumnos no logran entender el mensaje, o no son suficientemente estimulados para emprender el estudio personal.

El estilo de aprendizaje de los estudiantes de medicina chilenos ha sido evaluado, reportando que los estudiantes tienen preferencia por estilos de aprendizaje más reflexivos y teóricos y muy pocos con preferencia por el estilo pragmático y activo (Bitran et al., 2003; Díaz et al., 2009). Sin embargo, no existen reportes en nuestro país que analicen cuáles son los métodos de estudio de los alumnos de medicina. En la presente investigación analizamos qué métodos de estudio utilizan nuestros actuales alumnos de medicina durante los siete años de la carrera y los comparamos con los que usaron los profesores cuando fueron estudiantes.

\section{Materiales y métodos}

Evaluamos los métodos de estudio de los alumnos de la Escuela de Medicina de la Pontificia Universidad Católica (PUC) en diferentes momentos de la carrera, por medio de una metodología mixta: cuantitativa y cualitativa. El componente cuantitativo se logró mediante encuestas online ad hoc y aplicadas por primera vez en este estudio; el componente cualitativo mediante grupos focales. Los sujetos evaluados estaban al momento del estudio cursando quinto, sexto o séptimo año de la carrera además de un grupo de médicos recién egresados de la misma universidad. Adicionalmente evaluamos los métodos de estudios que usaron los Docentes de la Escuela de Medicina de la PUC, cuando ellos fueron estudiantes, mediante una encuesta online.

Encuestas: Por medio de una encuesta online a todos los alumnos de quinto año, luego de completar el curso de Obstetricia y Ginecología en los años 2010 y 2011, evaluamos los métodos de estudio utilizados por los alumnos durante el curso. En el 2010 formulamos 8 afirmaciones (Tabla 1), en una escala de Likert de 4 puntos: muy de acuerdo, bastante de acuerdo, poco de acuerdo y nada de acuerdo. En el 2011 presentamos 8 fuentes de información en el estudio personal (Tabla 2), y preguntamos la frecuencia de uso con otra escala de Likert de 4 puntos: siempre, casi siempre, rara vez y nunca. Los estudiantes sólo podían elegir una opción y debían responder todas las preguntas para completar la encuesta.

En el año 2014, efectuamos una encuesta online, a todos los alumnos que estuvieran cursando sexto o séptimo año de medicina (internado de cualquier especialidad) y los médicos recién egresados (terminaron medicina en diciembre del 2013). El objetivo de esta encuesta fue evaluar los métodos de estudio que habían utilizado a lo largo de toda la carrera. La encuesta contenía 12 preguntas cerradas (Tabla 3), todas de respuesta obligatoria. 
Por último, de forma cuantitativa, el 2014 se realizó una encuesta online a todos los docentes de la Escuela de Medicina con el fin de conocer los métodos de estudios que utilizaron cuando fueron estudiantes de medicina (Tabla 4).

Tabla 1: Encuesta realizada a alumnos de quinto año al final del curso de Obstetricia y Ginecología en el Año 2010.

1. Estudié de libros de textos y manuales

2. Me gustaría que existiera un texto guía del curso con todos los temas

3. El curso en general fue bueno, pero sería aún mejor si existiera un manual

4. Los apuntes de alumnos de años anteriores me fueron útiles

5. Los apuntes de mis compañeros me fueron útiles

6. Estudié principalmente de mis propios apuntes

7. Las diapositivas de las clases fue lo que más me ayudó a estudiar

8. Usé el sitio web del curso con frecuencia

Frente a cada una de las afirmaciones los alumnos debían elegir una de cuatro alternativas de respuesta: muy de acuerdo, bastante de acuerdo, poco de acuerdo y nada de acuerdo

Tabla 2: Encuesta sobre fuentes de estudio realizada a alumnos de quinto año al final del curso de Obstetricia y Ginecología en el Año 2011.

\begin{tabular}{|ll|}
\hline 1. & Clases (asistencia a clases) \\
\hline 2. & Diapositivas de clases \\
\hline 3. & Apuntes propios \\
\hline 4. & Apuntes de otros compañeros \\
\hline 5. & Apuntes de años anteriores \\
\hline 6. & Manual de Obstetricia y Ginecología \\
\hline 7. & Artículos científicos \\
\hline 8. & Libros de texto de Obstetricia y Ginecología \\
\hline
\end{tabular}

Respecto de estas ocho fuentes de estudio los alumnos debían indicar cuanto la usaron, teniendo como alternativas de respuesta: siempre, casi siempre, rara vez y nunca.

Grupos focales: el análisis cualitativo se logró mediante 2 grupos focales. El primero se realizó el año 2011, antes de comenzar el curso de Obstetricia y Ginecología. En esa oportunidad estudiamos dos grupos focales, de 10 alumnos cada uno, elegidos al azar de entre un total de 102 alumnos que cursaban $5^{\circ}$ año de Medicina. Les pedimos comentar libremente sobre sus métodos de estudio y específicamente el uso de: libros de texto, apuntes propios, apuntes de años anteriores, artículos científicos y diapositivas de clases. Les preguntamos cuáles estimaban como requisitos para un buen manual de curso y sobre la necesidad de tener un manual para cada curso. Recopilamos las opiniones en una grabación y representamos los resultados como una nube de palabras.
Tabla 3: Peguntas de encuesta online realizada en el año 2014 a alumnos de sexto, séptimo y recién egresados de la carrera de Medicina.

1. Enumera en orden de prioridad ( 1 el más importante, 6 el menos importante) ¿cuál fue tu fuente más importante de estudio personal entre 1-5 año de medicina? Respuestas posibles: apuntes de clases; diapositivas de clases; manual del curso; artículos científicos; libros de texto; google/internet.

2. Enumera en orden de prioridad ( 1 el más importante, 6 el menos importante) ¿cuál fue tu fuente más importante de estudio personal durante el internado? Respuestas posibles: apuntes de clases; diapositivas de clases; manual del curso; artículos científicos; libros de texto; google/internet.

3. No todos los cursos poseen un Manual del Curso, pero si existiera un Manual del Curso. ¿Sería esta tu principal fuente de estudio personal? Respuestas posibles: si - no

4. ¿Crees que todos los cursos deben tener un Manual para uso como texto guía? Respuestas posibles: si - no

5. ¿Crees que la existencia de un Manual del Curso facilita el aprendizaje? Respuestas posibles: si - no

6. Si el curso tiene un manual. ¿Qué formato prefieres? Respuestas posibles: digital (PDF o similar); impreso; online; otro (especificar)

7. Indica los que a tu juicio son requisitos para un buen Manual de Curso (elige las cosas que creas más importantes). Respuestas posibles: actualizado; esquemas-diagramas-algoritmos; bibliografía complementaria; complementario con las clases; tablas de resumen al final del capítulo; sistema de preguntas y respuestas.

8. Indica cuanto has usado el Manual de Ginecología y Obstetricia como fuente de estudio personal en los tres últimos años de la carrera. Respuestas posibles: mucho, bastante, poco, nada

9. En general durante tus años en la escuela de medicina, los sitios web de los cursos ¿fueron útiles? Respuestas posibles: si - no

10. Si los sitios web de los cursos tuvieran información actualizada, renovada e importante, ¿los usarías regularmente? Respuestas posibles: si - no

11. Durante la carrera (1-5 año), cuando la asistencia clases era voluntaria ¿Asistías regularmente a clases? Respuestas posibles: si-no

12. Durante la carrera de medicina. Crees que las clases debiesen ser: presenciales, online o ambas. Debe elegir solo una opción.

Tabla 4: Preguntas de encuesta online realizada en el año 2014 a docentes de la Escuela de Medicina.

1. ¿En qué año naciste?

2. Enumera en orden de prioridad ( 1 el más importante, 6 el menos importante) cuál fue tu fuente más importante de estudio personal entre 1-5 año de medicina. Respuestas posibles: apuntes de clases; diapositivas de clases; manual del curso; artículos científicos; libros de texto; google/internet.

3. Enumera en orden de prioridad ( 1 el más importante, 6 el menos importante) cuál fue tu fuente más importante de estudio personal en el internado. Respuestas posibles: apuntes de clases; diapositivas de clases; manual del curso; artículos científicos; libros de texto; google/internet.

4. Durante la carrera (1-5 año), cuando la asistencia a clases era voluntaria asistías regularmente a clases. Respuestas posibles: si - no

5. En la época actual las clases de medicina debieran ser: presenciales, online o ambas. Debe elegir solo una opción.

6. En la época actual ¿Crees que todos los cursos de la escuela de medicina entre 1-5 deben tener un manual para uso como texto guía? Respuestas posibles: si-no

7. ¿Crees que la existencia de un Manual del Curso facilita el aprendizaje? Respuestas posibles: $\mathrm{si}$ - no 
El segundo grupo focal se realizó en el 2015. Fue una entrevista con estudiantes que cursaban $6^{\circ}$ año de Medicina, que iniciaron la rotación obligatoria de la especialidad Obstetricia y Ginecología, quienes habían efectuado el curso de Obstetricia y Ginecología durante el año anterior ( $5^{\circ}$ año). Los participantes del grupo focal fueron invitados en forma personal considerando un muestreo opinático o intencionado que garantizara la presencia en el grupo de hombres y mujeres. El grupo quedó constituido por cuatro hombres y cuatro mujeres que cumplen con el criterio muestral. Las categorías analizadas se describen en la Tabla 5.

Tabla 5: Categorías analizadas Grupo Focal Mayo 2015.

1. Clasificación general de métodos de estudio utilizados por los estudiantes

2. Estrategias de aprendizaje empeladas hasta quinto año

3. Estrategias de aprendizaje empleadas en sexto año

4. Opinión sobre resúmenes elaborados por compañeros

5. Formato de manual virtual de obstetricia y ginecología

6. Relación entre manual virtual de obstetricia y ginecología versus clases de quinto año

7. Relación entre manual virtual de obstetricia y ginecología versus seminarios de sexto año

Para todos los grupos focales, se llevaron a cabo los procedimientos éticos relativos a este tipo de pesquisas firmando cada participante un Consentimiento Informado, que da cuenta del uso que se hará de la información, las consideraciones de confidencialidad que la resguardan y la autorización que otorgan para emplearla en los términos allí formulados. Una copia de ese documento quedó en poder del participante y otra en poder del equipo de investigación. Para ambos grupos focales se empleó el análisis de contenido para llegar a conformar las categorías, apoyándose en elementos basados en la Teoría Fundada (Strauss \& Corbin, 2002) y en el enfoque más tradicional de Hernández, Fernández y Baptista (Hernández et al., 2006). Se realizó una categorización abierta de los conceptos obtenidos y la información recogida se trianguló con la participación de dos analistas y con el apoyo de la herramienta computacional atlas.ti. Las encuestas fueron respondidas de forma anónima y los resultados de los grupos focales fueron confidenciales.

Análisis estadístico: los datos se muestran como porcentaje de respuestas, expresados de modo dicotómico. Sobre los datos obtenidos se aplicó la prueba de chi cuadrado con corrección de yates; un valor $p<0.05$ fue considerado significativo.

\section{Resultados}

Encuestas online 2010 y 2011: La encuesta del año 2010 se envió a 106 alumnos de quinto año, de los cuales 83 la respondieron (78\%). Los resultados se presentan en la Figura 1. Destaca que el $80 \%$ de los alumnos refiere no haber hecho uso de libros de texto y que el $98 \%$ consideró que, aunque el curso es muy bueno, la presencia de un manual haría aún mejor el curso.

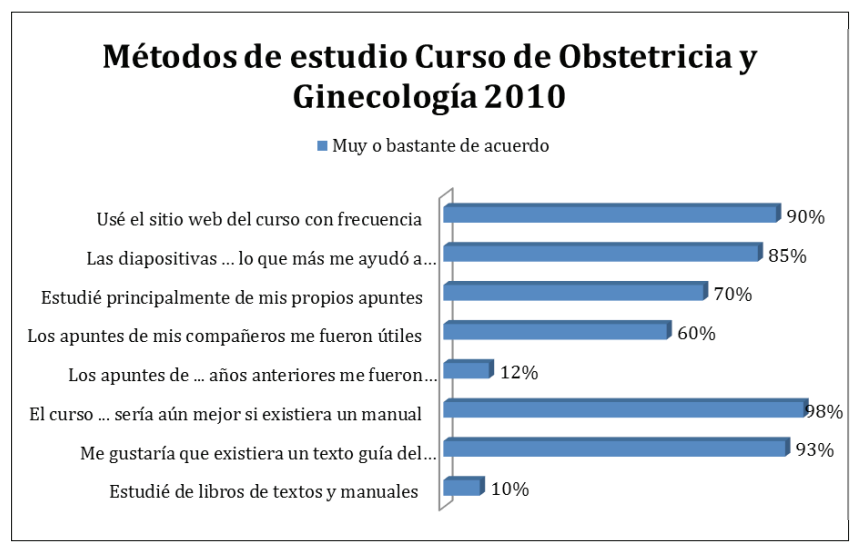

Figura 1: Respuesta de encuesta realizada a alumnos de quinto año al final del curso de Obstetricia y Ginecología en el Año 2010.

La encuesta online realizada el año 2011 fue respondida por 71 de 102 alumnos (70\%), sus resultados se muestran en la Figura 2. Observamos que el $99 \%$ de los alumnos nunca o rara vez hizo uso de libros de texto; y que la gran mayoría hizo uso de los artículos científicos ("papers") y del Manual de Obstetricia y Ginecología (manual propio del curso que fue publicado ese año, pero cuya lectura no era obligatoria).

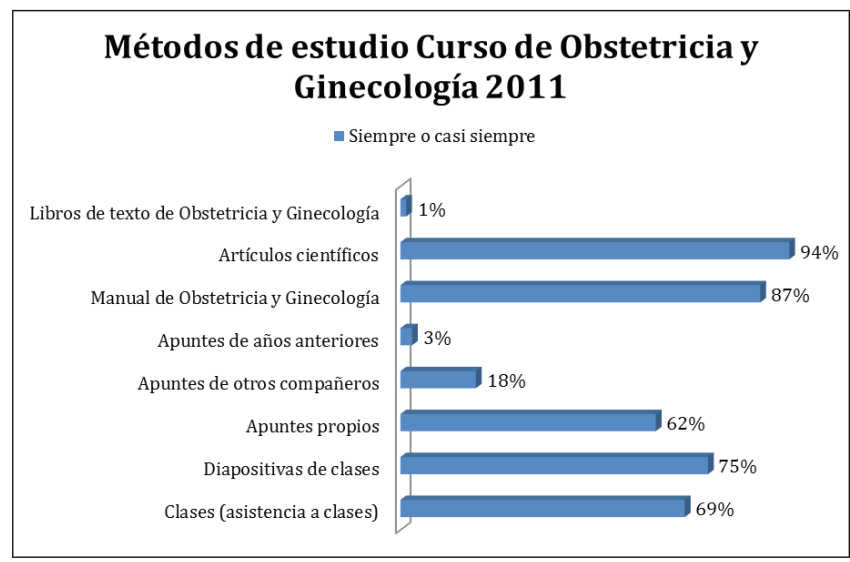

Figura 2: Respuesta de encuesta sobre fuentes de estudio realizada a alumnos de quinto año al final del curso de Obstetricia y Ginecología en el Año 2011. 
Ralph et al.

Grupo Focal 2011: Como se observa en la nube de palabras obtenida del grupo focal del año 2011 (Figura 3), los métodos de estudio que más utilizan los alumnos son los apuntes de clases, el manual de la asignatura, y la asistencia a clases. En relación a los apuntes de años anteriores consideran que son inútiles, y que en general están desactualizados y con muchos errores.

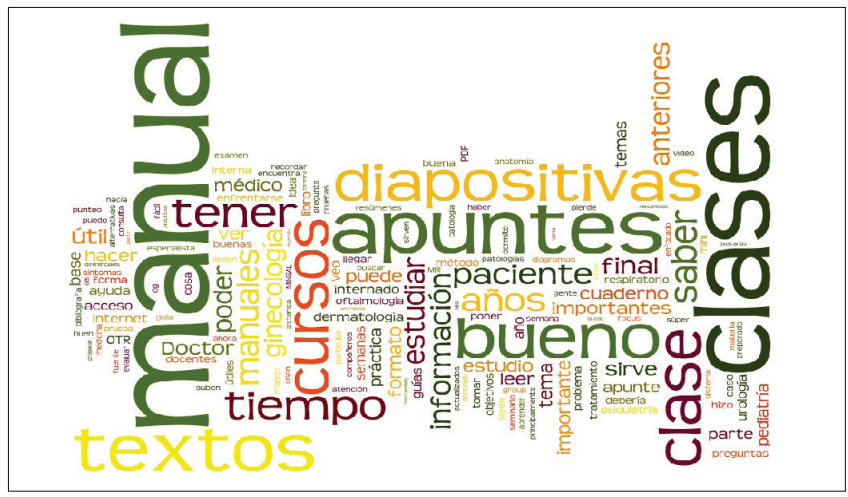

Figura 3: Nube de palabras representativa del grupo focal efectuado antes de curso de obstetricia y ginecología en 2011.

Encuesta online 2014: La encuesta online a estudiantes del año 2014 fue enviada a un total de 300 sujetos; de éstos, 150 tuvieron acceso a la encuesta, y 139 (92\%) la contestó. Observamos diferencias en las fuentes de estudio entre los cursos de primero a quinto año en comparación con los alumnos de sexto y séptimo año (Internado) de Medicina (Figura 4 y 5). Para el grupo de primero a quinto año (Figura 4) su primera fuente de estudio fueron los apuntes de clases, seguido de la asistencia a clases y los manuales. Por el contrario, durante el internado, la principal fuente de estudio fue uso de un manual, seguido de apuntes de clases y artículos científicos.

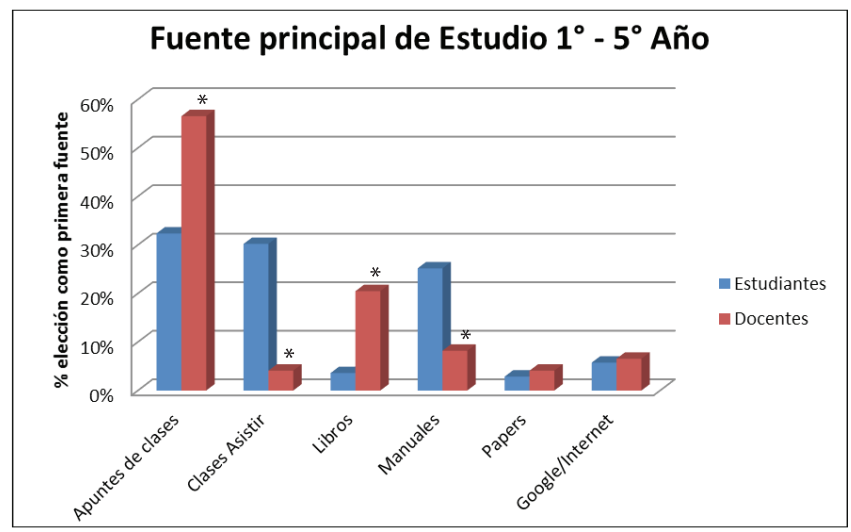

Figura 4: Encuesta online para evaluar fuentes de estudio durante la Carrera de Medicina en los primeros 5 años. ${ }^{*}=p<0.05$ docentes vs estudiantes.

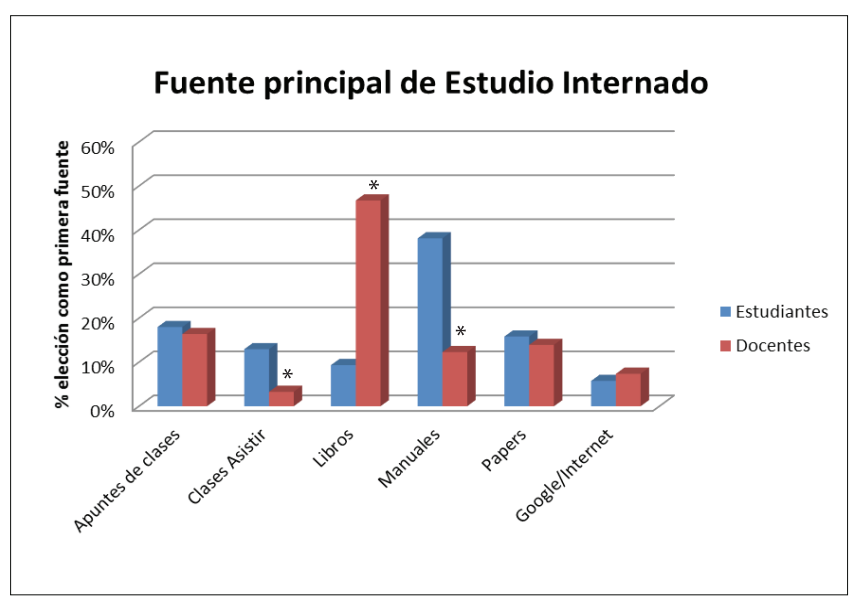

Figura 5: Encuesta online para evaluar fuentes de estudio durante el Internado. ${ }^{*}=p<0.05$ docentes vs estudiantes.

La encuesta online a docentes se envió a 600 sujetos, de los cuales 122 contestaron la encuesta (17\%). Entre los docentes que respondieron la encuesta, 43\% había nacido entre 19451964 y 57\% entre 1965-1981. Tanto para el primer grupo, que corresponde a los baby boomers, como para el segundo grupo, la llamada generación $X$, lo más importante en orden de prioridad, en cuanto a métodos de estudio de primero a quinto año de medicina, fueron los apuntes de clases, seguido por los libros de texto; en general indicaron que lo menos importante fue el uso de artículos científicos y Google/Internet. En relación a los métodos de estudio utilizados durante el internado, ambas generaciones consideraron que lo más importante fueron los libros de texto. La generación de los baby boomers refiere en segundo lugar como método de estudio los apuntes de clases; a diferencia de la generación $X$ que en segundo lugar indica el uso de artículos científicos (Figura 6), aunque estas diferencias no fueron significativas.

Las Figuras 4 y 5 muestran la comparación entre los métodos de estudio usados por los estudiantes en comparación con los métodos usados por los profesores. Durante los años de primero a quinto, destaca el uso significativamente mayor por los profesores de los apuntes de clases y los libros, mientras que los alumnos hicieron uso significativamente mayor de asistencia a clases y manuales (Figura 4). De modo similar, durante el internado, los profesores usaron de modo significativamente mayor, los libros como fuente principal de estudio; mientras que los estudiantes prefirieron los manuales (Figura 5). 


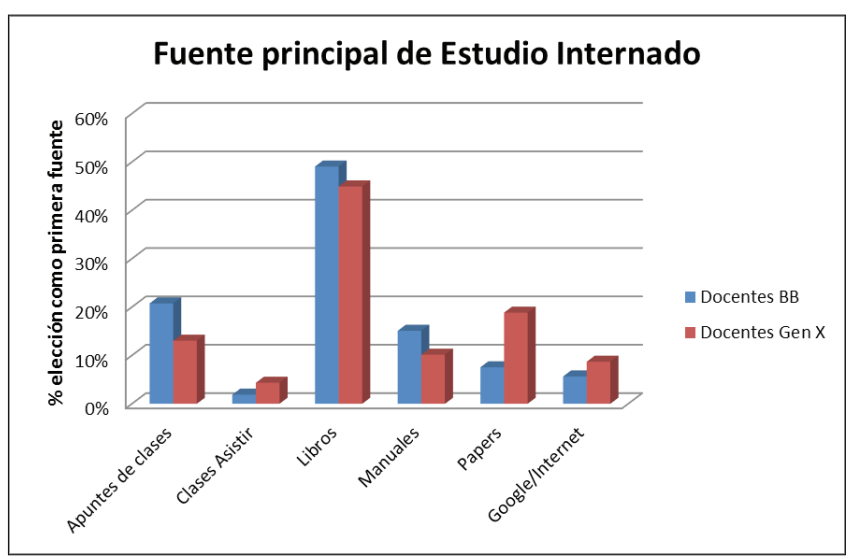

Figura 6: Encuesta online para evaluar fuentes de estudio durante el Internado, comparando docentes de diferentes edades. Docentes BB = docentes de la generación baby boomers (nacidos entre 1945 y 1964). Docentes Gen X = docentes de la generación X (nacidos entre 1965 y 1981).

Considerando que no todos los cursos cuentan con un manual, les preguntamos a los estudiantes su opinión en relación a su uso, en caso de estar siempre disponible; el $90 \%$ indicó que haría uso de un manual como fuente principal de estudio si el curso lo tuviera. El $90 \%$ de los encuestados respondió afirmativamente a la pregunta ¿Crees que todos los cursos deben tener un Manual para uso de texto guía? El 98\% responde que sí a la pregunta ¿Crees que la existencia de un Manual del Curso facilita el aprendizaje?

Por el contrario, la mayoría de los docentes (54\%) considera que no todos los cursos de la carrera de medicina debieran tener un Manual de uso como texto guía. Sin embargo, la mayoría de ellos (86\%) considera que la presencia de un Manual del curso facilitaría el aprendizaje de los alumnos.

Respecto al formato preferido para un Manual del Curso, un 76\% de los estudiantes prefirió el formato digital para usar en su tableta o computador (Figura 7) y la gran mayoría considero que uno de los requisitos más importantes era que el manual estuviera actualizado (Figura 8).

En relación al uso del Manual del curso de Obstetricia y Ginecología como fuente de estudio en los tres últimos años de la carrera, para estudiantes y egresados que respondieron la encuesta, su uso fue muy importante en quinto y sexto año, pero poco en séptimo año (Figura 9).

Un 45\% consideró inútil el uso de sitios web de los cursos durante la carrera de medicina, sin embargo, cuando les preguntamos si ellos harían uso de éstos en caso de que tuviera información actualizada, renovada e importante, el $98 \%$ del total de los alumnos dijo que los usaría.
Si el curso tiene un manual, ¿qué formato prefieres?

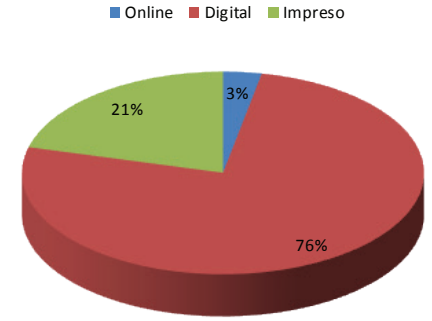

Figura 7: Encuesta online entre estudiantes y médicos recién egresados para evaluar el formato preferido de un manual.

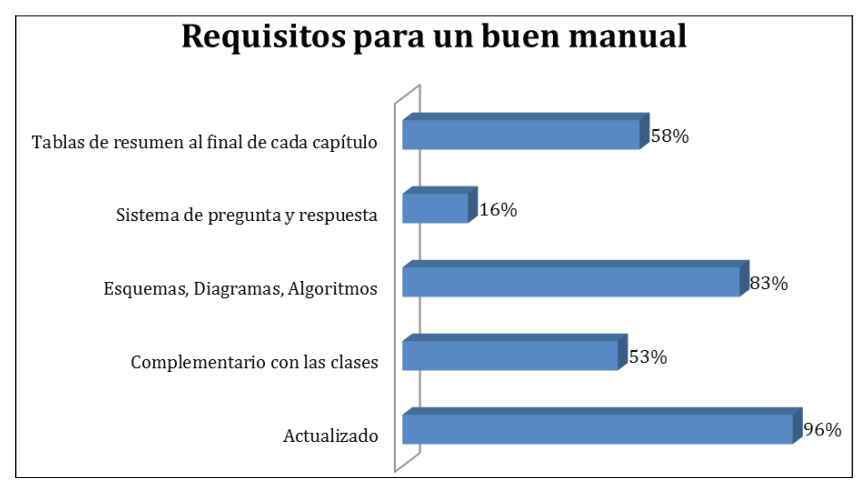

Figura 8: Encuesta online entre estudiantes y médicos recién egresados para evaluar características preferidas de un manual.

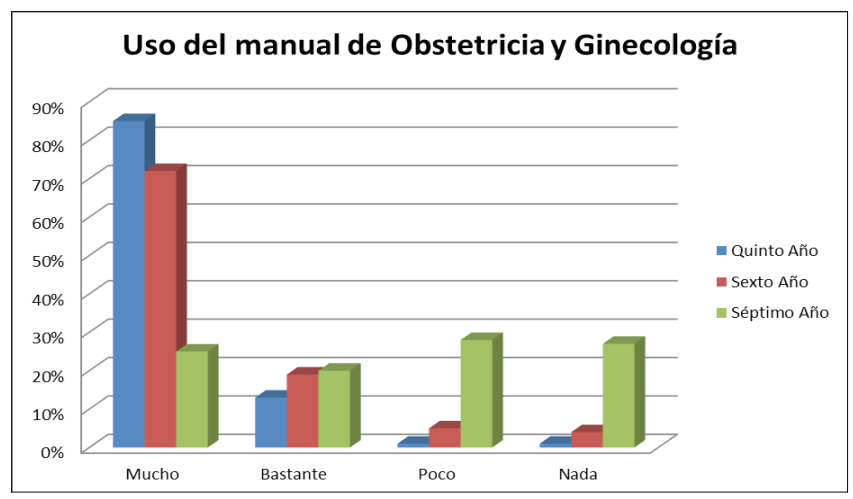

Figura 9: Resultados encuesta en línea a estudiantes y egresados respecto al uso de Manual de Ginecología y Obstetricia como fuente de estudio personal en los últimos años de la carrera.

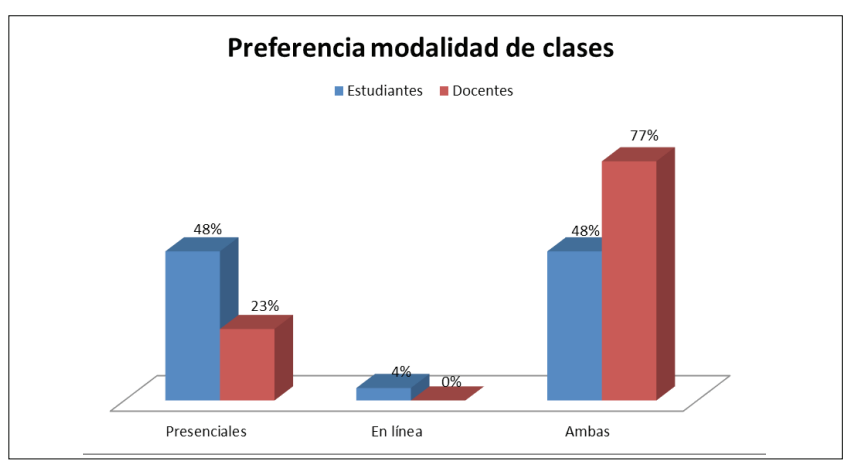

Figura 10: Resultados encuesta en línea a estudiantes y egresados indicando su preferencia respecto de la modalidad de clases. 
Como se observa en la Figura 10, casi la mitad de los estudiantes prefieren que las clases sean siempre presenciales, mientras que la otra mitad prefiere que las clases sean tanto presenciales como en línea. Por el contrario, la mayoría de los docentes considero que actualmente las clases debieran ser presenciales y en línea.

El $81 \%$ de los alumnos refirió que sí asistió a clases durante la carrera (1-5 año) cuando las clases eran voluntarias. Los docentes de medicina indicaron que asistían regularmente a clases (95\%) durante los cursos de primero a quinto aun cuando la asistencia a clases era voluntaria.

Grupo Focal mayo 2015: El análisis se efectuó de acuerdo a las categorías contenidas en la Tabla 5. En general los alumnos de medicina consideran que los métodos de estudio pueden ser individuales o grupales; y metodológicamente a través de lectura, visuales y/o auditivos. Como estrategia de aprendizaje durante los primeros 5 años de la carrera, lo más importante eran los resúmenes y los textos guías como manuales del curso. Pero luego, en el internado, necesitan de una metodología más práctica, por ejemplo, seminarios; en esta etapa el manual o los apuntes quedan como base de apoyo. Consideran que durante la carrera de medicina tener un manual específico del curso les permite tener la información básica necesaria agrupada toda en un solo texto.

\section{Discusión}

Los resultados cuantitativos y cualitativos de esta investigación, nos han permitido detectar que los estudiantes de medicina de la Pontificia Universidad Católica de Chile, consideran muy útiles los manuales como fuentes de estudio durante la carrera de medicina, prefieren para ellos un formato electrónico, y es muy importante que estos manuales estén actualizados. Los estudiantes incluidos en esta investigación refieren que su asistencia a clases es buena y la mitad de ellos prefiere que las clases sean solo presenciales. Los docentes encuestados refieren haber hecho uso prioritario de los libros de texto durante sus estudios de medicina; indican que su asistencia a clases fue mayoritaria, y piensan que en nuestros días, las clases debieran ser en metodología mixta (presencial y en línea). En esta investigación se evaluaron por medio de distintas metodologías los métodos de estudio utilizados por distintas generaciones de alumnos y docentes de medicina UC: grupos focales y encuestas online. La metodología de los grupos focales fue la adecuada para desarrollar una teoría fundamentada, mientras que las encuestas permitieron conocer de modo cuantitativo las observaciones.

El uso de los conceptos Manual ("handbook") o Libros ("textbook) no está claramente definido en la literatura médica; pero si existe una clara diferenciación en nuestro uso habitual. El manual es un texto, habitualmente breve, cuyo contenido está organizado de modo simple, y se ajusta con precisión a los contenidos propios de la materia en estudio. El libro, sea impreso o digital, corresponde habitualmente a una obra de mayor extensión, en que el autor resume contenidos de varias fuentes, indicando los pro y contras de cada aseveración, y no llegando necesariamente a un resumen de su opinión personal; habitualmente los libros no están pensados para servir a un solo ramo de estudio, sino que a consultas como guía desde diferentes ramos o carreras.

Con este concepto en mente, pudimos observar que los libros de texto han perdido importancia como fuente de información entre los estudiantes de medicina UC y que lo que los alumnos actuales consideran de mayor utilidad como fuentes de estudio es el manual del curso. Es importante notar que solo un 70-78\% de los alumnos encuestados respondió la encuesta en línea en que se pregunta por el uso de los textos de estudios; la metodología empleada hace imposible saber si quienes no respondieron, pudieron haber preferido los textos por sobre otras fuentes. Por el contrario, quienes hoy en día son docentes de la escuela de medicina, usaron como principal fuente de estudio a los libros, cuando ellos fueron estudiantes. Esta diferencia tensiona la relación docente/estudiante, pues muestra la necesidad de generar manuales que permitan a los alumnos estudiar conforme a sus necesidades aun cuando los docentes no creen que esto sea necesario.

Probablemente los estudiantes de medicina UC actuales prefieren textos con contenidos acotados al curso y actualizados; y lamentablemente lo clásicos textos de estudio no cumplen, habitualmente, con estos requisitos. Los estudiantes acceden a distintos sistemas tecnológicos con facilidad, hecho que explicaría por qué en vez de portar grandes libros prefieren hacer uso de sus tabletas o computadores para buscar la información que necesitan (Kunakov, 2011). Adicionalmente, a los estudiantes de hoy les interesa optimizar su tiempo y cuidar su tiempo libre, prefiriendo por ello textos con contenidos esenciales y revisados por los mismo profesores que dictan las clases; el resto de la información, más específica o profunda, podrán obtenerla desde sus casas en artículos científicos (Popescu \& Navarro, 2005).

Pese a que al encuestar a los docentes de medicina UC ellos refieren que no es necesaria la existencia de manuales para cada curso, sí piensan que la existencia de un Manual del Curso facilitaría el aprendizaje, opinión que es compartida por los alumnos actuales. En ocasiones los profesores no aprecian que el avance de los medios de comunicación y tecnológicos ha hecho que los estudiantes sean diferentes; tienen miedo al cambio y es por esto que siguen creyendo que los métodos de estudio que ellos usaron son los mejores. La aparición de los manuales constituye una amenaza a sus clásicos 
métodos de enseñanza, sin embargo tarde o temprano tendrán que adaptarse a los intereses e inquietudes de los "nativos digitales" (Kunakov, 2011).

Los alumnos que efectuaron el curso de Obstetricia y Ginecología en el año 2010 en nuestra Escuela de Medicina UC indicaron que sería muy importante contar con un manual; en respuesta a lo cual, en el año 2011 se publicó el Manual de Obstetricia y Ginecología. En el 2011 los alumnos hicieron uso frecuente del manual aun cuando su lectura no era obligatoria. El manual también fue fuente principal de estudio en el internado de sexto año, pero no en séptimo año. La diferencia se explica probablemente pues el internado de sexto año de obstetricia y ginecología es obligatorio, mientras que el internado de séptimo año no tiene pasantías obligatorias de obstetricia y ginecología. Otra explicación posible es que en séptimo año los alumnos ya han alcanzado los conocimientos mínimos y prefieren el uso de otros medios más específicos, como los artículos científicos y seminarios relacionado con temas de la especialidad. Los artículos científicos están en tercer lugar como fuente de estudio para los internos de sexto y séptimo años de medicina UC, probablemente reflejando el estímulo por parte de los docentes para la lectura de artículos específicos y actualizados de las especialidades en que estén rotando; al final de la carrera los alumnos presentan más destrezas en las técnicas de búsqueda de información y por lo tanto les resulta fácil el acceso a esta fuente de información. Durante los cursos de 1-5 año, los artículos científicos son mencionados como pocos utilizados (Figura 4). Esto contrasta con las encuestas online para los cursos de quinto años de 2010 y 2011 en que los alumnos hicieron uso muy importante de los artículos científicos. La explicación para esta discordancia es que en esos dos años, la lectura de artículos científicos era obligatoria y de evaluación diaria. Esto nos indica que si bien los artículos científicos no son frecuentemente usados por los alumnos antes del internado, su uso si puede ser correctamente estimulado.

Es interesante destacar la importancia que tienen los apuntes de las clases como fuente de estudio, tanto en las generaciones actuales como en los docentes cuando ellos eran alumnos de medicina. La gran mayoría de los alumnos asistía a clases, de donde provienen esos apuntes sin duda, ya que dijeron no usar apuntes de años anteriores o de sus compañeros. Sin embargo, creemos que estas clases que refieren preferir los alumnos, no son las antiguas clases de larga duración destinadas solo a la transmisión del conocimiento. Las clases que hoy deben efectuarse son clases que usen metodologías activas de aprendizaje, como es el caso de las clases invertidas, las tecleras, etc.

La mitad de los estudiantes de medicina UC prefiere clases solo presenciales, y la otra mitad una modalidad mixta. Probablemente lo que vemos en estos resultados es un reflejo de la transición en el grupo de estudiantes, fines de la generación $Y$ e inicio de la generación $Z$. Por lo tanto, es esperable que cada vez sea más frecuente ver grupos de estudiantes que prefieran mezclar las clases online con las presenciales, dado que eso les permitirá organizar mejor su tiempo y disfrutar más de sus espacios libres (Han et al., 2014). No negamos la importancia del contacto docente/alumno; sin embargo, ya existen varios estudios que muestran la buena adherencia al sistema de educación online (McKimm et al., 2003). Estimamos que, ante los cambios sustanciales en los intereses de los alumnos, es importante capacitar a los académicos y docentes en el uso de las nuevas tecnologías de la información y educación, mezclando modelos presenciales con aquellos en línea, de modo armónico.

En este contexto es interesante lo observado al encuestar a los docentes y preguntarles sobre su opinión sobre la modalidad que debieran tener las clases; a diferencia de lo que habríamos esperado, la gran mayoría considera que en la época actual las clases debieran ser presenciales y en línea. Probablemente esta opinión demuestra que nuestros docentes han llegado a comprender las características y necesidades de los actuales estudiantes; un hecho que genera confianza en que aparecerán cambios positivos de la metodología docente. El uso de internet mejoraría el aprendizaje de los alumnos (McKimm et al., 2003; Hernández et al., 2006), y esto debe ser reconocido y usado por los docentes.

Es llamativo el poco uso que los alumnos hacen de los sitios web de los cursos, considerando su acceso preferencial a la experiencia online. Estimamos, sin embargo, que esto se debe a que los profesores hacen poco uso de los sitios web de los cursos para compartir información relevante con los alumnos. En efecto, la gran mayoría indicó que si usaría el sitio web del curso si este tuviera información relevante y actualizada.

Es un hecho que los estudiantes que hoy ingresan a estudiar medicina son diferentes de los que ingresaban hace años, y que los nuevos estudiantes poseen características generacionales que les son propias. Sin embargo, es imposible mediante esta investigación determinar si solo este hecho explica las diferencias detectadas, dejando de lado el rol que le cabe a los docentes en cuanto a la determinación de algunos comportamientos; es posible que cambios en la malla curricular, comportamiento del cuerpo docente, políticas institucionales y nuevas metodología de enseñanza/aprendizaje, sean causa (al menos parcial) y no consecuencia de los cambios de los estudiantes. Es así como desde el año 2000, la Escuela de Medicina UC implementó políticas de desarrollo docente, plasmadas principalmente en el Diplomado en Docencia, que los académicos deben completar, y que les permite adquirir e integrar en su práctica metodologías de enseñanza centradas en el estudiante. 
Se deduce que es indispensable que los docentes conozcan y comprendan a los nuevos estudiantes, y que los cursos se adapten a las nuevas características, no como un modelo servicial, sino que como una obligación para lograr mejores médicos. En este procesos será indispensable la capacitación docente incluyendo programas de acuerdo a las competencias (Cumming \& Ross, 2007), uso de metodología de enseñanza y aprendizaje activo (Riquelme et al., 2011), enseñanza a distancia y en pequeños grupos, mentorías y retroalimentación efectiva (Bitran et al., 2010). Será necesario facilitar el proceso de adecuación docente, preparado programas y guías de estudio, retroalimentación, consejería, mentorías, permisos y financiamiento para asistir a congresos, regulación de las horas de trabajo, tiempo protegido para las instancias académicas, entrenamiento y evaluación de las destrezas clínicas (Herrera et al., 2013).

\section{Conclusión}

El cambio generacional en los alumnos de medicina UC es evidente, este cambio explica diferentes motivaciones y por supuesto técnicas de estudio/aprendizaje. Es indispensable que los métodos docentes se adapten a estas nuevas características, y en este proceso debemos acompañar a los profesores; colaborando con ellos a buscar nuevas herramientas que le permitan adaptarse a los nuevos intereses y métodos de estudio de sus alumnos.

\section{Referencias}

Bitran C M, Zúñiga P D, Lafuente G M, Viviani G P \& Mena C B. (2003). Tipos psicológicos y estilos de aprendizaje de los estudiantes que ingresan a Medicina en la Pontificia Universidad Católica de Chile. Revista medica de Chile 131, 1067-1078.

Bitran M, Mena B, Riquelme A, Padilla O, Sanchez I \& Moreno R. (2010). An instrument in Spanish to evaluate the performance of clinical teachers by students. Revista medica de Chile 138, 685-693.

Cumming A \& Ross M. (2007). The Tuning Project for Medicine--learning outcomes for undergraduate medical education in Europe. Medical teacher 29, 636-641.

Daura F. (2011). Las estrategias docentes al servicio del desarrollo del aprendizaje autorregulado. Estudios Pedagógicos 37, 77-88.

Díaz-Véliz G, Mora S, La Fuente-Sánchez JV, Gargiulo PA, Bianchi R, Terán C, Gorena D, Arce J \& Escanero-MArcen JF. (2009). Estilos de aprendizaje de estudiantes de medicina en universidades latinoamericanas y españolas: relación con los contextos geográficos y curriculares. Educación Médica 12, 183-194.
Han H, Nelson E \& Wetter N. (2014). Medical students online learning technology needs. The clinical teacher 11, 15-19.

Hernández R, Fernández C \& Baptista P. (2006). Metodología de Ia Investigación. Cuarta Edición. McGraw-Hill, Iztapalapa. Mexico.

Herrera CA, Niklitschek I, Pizarro M, Solis N, Olivos T, Rojas V, Etcheberry L, Rivera H, Munoz E, Bitran M, Padilla O \& Riquelme A. (2013). Identifying the main training needs of postgraduate medical program managers. Revista medica de Chile 141, 1126-1135.

Kunakov N. (2011). Medical schools: students today. Revista medica de Chile 139, 524-528.

McKimm J, Jollie C \& Cantillon P. (2003). ABC of learning and teaching. Web based learning. Bmj 326, 870-873.

Money SR, O'Donnell ME \& Gray RJ. (2014). In the time of significant generational diversity - surgical leadership must step up! The surgeon. Journal of the Royal Colleges of Surgeons of Edinburgh and Ireland 12, 3-6.

Ponce-De León ME. (2014). Tendencias actuales en la enzeñanza de la Medicina. Estrategias del aprendizaje en medicina. Gaceta Médica de México 140, 305-306.

Popescu BM \& Navarro V. (2005). Comparación del aprendizaje en internet con la clase convencional en estudiantes de medicina, en Argentina. Educación Médica 8, 28-31.

Prensky M. (2001). Digital Natives, Digital Immigrants Part 1. On the Horizon 9, 1-6.

Riquelme A, Mendez B, de la Fuente P, Padilla O, Benaglio C, Sirhan M \& Labarca J. (2011). Development and validation of a questionnaire on perception of portfolio by undergraduate medical students. Revista medica de Chile 139, 45-53.

Silva-Peña I, Borrero AM, Marchant P, González G \& Novoa D. (2006). Percepciones de jóvenes acerca del uso de las tecnologías de información en el ámbito escolar. Ultima década 14, 37-60. Strauss A \& Corbin J. (2002). Bases de la investigación cualitativa. Técnicas y procedimientos para desarrollar la teoría fundamentada. Ediorial Universidad de Antioquía, Antioquía, Colombia.

\section{Nota: *}

Tabula rasa: tabla rasa se refiere a la antigua tesis epistemológica de que los individuos nacen con la mente vacía, haciendo necesario que todos los conocimientos sean introducidos en la mente a través de proceso de enseñanza/aprendizaje. Se transforma en lo contrario de la enseñanza activa que hoy promovemos. 